\title{
"Multivariate count data generalized linear models: Three approaches based on the Sarmanov distribution"
}




\section{$\boxplus|R| E|A|$}

Institut de Recerca en Economia Aplicada Regional i Pública

Research Institute of Applied Economics

\section{Universitat de Barcelona}

Av. Diagonal, $690 \bullet 08034$ Barcelona

WEBSITE: www.ub.edu/irea/•CONTACT: irea@ub.edu

The Research Institute of Applied Economics (IREA) in Barcelona was founded in 2005, as a research institute in applied economics. Three consolidated research groups make up the institute: AQR, RISK and GiM, and a large number of members are involved in the Institute. IREA focuses on four priority lines of investigation: (i) the quantitative study of regional and urban economic activity and analysis of regional and local economic policies, (ii) study of public economic activity in markets, particularly in the fields of empirical evaluation of privatization, the regulation and competition in the markets of public services using state of industrial economy, (iii) risk analysis in finance and insurance, and (iv) the development of micro and macro econometrics applied for the analysis of economic activity, particularly for quantitative evaluation of public policies.

IREA Working Papers often represent preliminary work and are circulated to encourage discussion. Citation of such a paper should account for its provisional character. For that reason, IREA Working Papers may not be reproduced or distributed without the written consent of the author. A revised version may be available directly from the author.

Any opinions expressed here are those of the author(s) and not those of IREA. Research published in this series may include views on policy, but the institute itself takes no institutional policy positions. 
Starting from the question: "What is the accident risk of an insured?", this paper considers a multivariate approach by taking into account three types of accident risks and the possible dependence between them. Driven by a real data set, we propose three trivariate Sarmanov distributions with generalized linear models (GLMs) for marginals and incorporate various individual characteristics of the policyholders by means of explanatory variables. Since the data set was collected over a longer time period (10 years), we also added each individual's exposure to risk. To estimate the parameters of the three Sarmanov distributions, we analyze a pseudomaximumlikelihood method. Finally, the three models are compared numerically with the simpler trivariate Negative Binomial GLM.

\section{JEL classification: C51, G22}

Keywords: Multivariate counting distribution, Sarmanov distribution, Negative Binomial distribution, Generalized Linear Model, ML estimation algorithm.

Catalina Bolancé: Risckcenter Research group-IREA. Av. Diagonal 696; 08034 Barcelona ,Spain. E-mail: bolance@ub.edu

Raluca Vernic: Faculty of Mathematics and Informatics Ovidius University of Constanta; Bd Mamaia 124, 900527 Constanta, Romania. E-mail: rvernic@univ-ovidius.ro

\section{Acknowledgements}

Catalina Bolance' acknowledges the Spanish Ministry of Education and the ERDF for grant ECO2016-76203-C2-2. 


\section{Introduction}

Quantifying the risk of an accident is essential for pricing in insurance markets. Insurers tended to focus on the risk associated with the policy contracted during a certain period, typically one year in non-life lines. Indeed, various studies adopt this approach when dealing with pricing in auto insurance lines (see, for example, Abdallah et al., 2016; Boucher and Inoussa, 2014; Bolancé et al., 2008; Boucher et al., 2007; Bolancé et al., 2003; Pinquet et al., 2001). However, few papers to date have attempted to analyse the policyholder's accident risk from a multivariate perspective. Two examples of the use of bivariate count data models to tackle pricing in the auto insurance line are provided by Abdallah et al. (2016) and Bermudez and Karlis (2011), while Shi and Valdez (2014) use copula-based models to a trivariate analysis in this same line.

Therefore, we seek to address the following question: What is the client's accident risk when he has more than one type of coverage from his insurance company? This question could be answered by using univariate generalized linear models (GLMs), i.e., we could estimate one model for each coverage assuming the independent behavior of this policyholder in relation to each coverage. Alternatively, we can use a multivariate GLM that allows us to obtain a joint distribution associated with each individual, which also takes into account the fact that the risks of accident covered by the insurance company are dependent.

In this paper, we analyze different multivariate models for claims frequencies with GLMs for marginals, and we propose three multivariate GLMs based on the Sarmanov distribution, on the grounds that they are better alternatives to the multivariate Negative Binomial (NB) model. The multivariate models proposed allow us to fit the multivariate accident rate of the policyholders that have contracted different risk coverages associated with different policies in distinct non-life lines. Our aim is to capture the relationship between the behavior of a policyholder as regards the different risk coverages he has contracted. We show that the approaches based on the Sarmanov distribution allow us to model the dependence under different assumptions, i.e. we can directly assume that the dependence exists between the number of claims in each coverage and, alternatively, we can assume that the dependence exists between the random effects associated with unobserved factors.

Taking the global perspective of the client of an insurance company, some analyses have attempted to consider the information contained in the different policies of the same policyholder (representing different insurance coverages) with regards to the policyholder's profitability and loyalty. For example, Guelman and Guillén (2014) analyzed the price elasticity associated with an insurance contract maximizing the overall profitability of the policyholder (see also, Guelman et al., 2014). Other analyses have focused on the policyholder lapse as they study the relationship that exists between the cancellations of different policies by the same policyholder (see, e.g., Guillén et al., 2012; Brockett et al., 2008).

To have sufficient multivariate information about the policyholders' behavior, we need to observe this behaviour over a long period, i.e., more than one year. Typically, insur- 
ance companies use annual information for pricing in non-life insurance lines. However, insurance premiums are subject to different adjustments, some of which may be related to the risk quantification and others to marketing strategies or customer selection. Thus, insurers need to analyze portfolio information over several periods and so, here we analyze 10 years of claims information on auto and home lines.

Univariate mixed Poisson GLMs have been widely used in non-life insurance pricing (see Frees, 2009, Chapter 12, for a review). In this paper, we study three trivariate GLMs based on the trivariate Sarmanov distribution, and we use them to model trivariate count data corresponding to frequency of claims in non-life insurance. The first model consists of the discrete trivariate Sarmanov with NB GLM marginals. The second model is similar to that proposed by Abdallah et al. (2016) for the bivariate case, although it incorporates certain modification. Thus, we mix the trivariate model of independent Poisson distributions with a trivariate Sarmanov distribution with Gamma distributed marginals. In the third model, we mix a discrete trivariate Sarmanov distribution with Poisson marginals with three independent Gamma distributions. The main difference between these models lies in their respective dependence structures. In all three we assume that each policyholder has a given exposure to risk which can differ for the distinct insurance lines. Moreover, the expected number of claims associated with the analyzed risks depends on a set of explanatory variables. In our case, these explanatory variables are related to the customer's characteristics and are the same for each counting variable, but they can change in function of the analyzed risk.

The maximum likelihood (ML) estimation of all the parameters of a model based on the trivariate Sarmanov distribution is far from straightforward. It requires adding different restrictions to the parameters and an optimal solution is not readily found. Alternatively, we analyze a pseudo-maximum-likelihood estimation method based on a conditional likelihood that allows us to estimate all three trivariate models obtained from the Sarmanov distribution.

We also compare the three Sarmanov's distributions with the well known alternative multivariate Poisson GLM mixed with Gamma that is, with the trivariate NB GLM. The numerical study is conducted on a set of trivariate claims data from auto and home insurance lines, collected over a period of 10 years from a portfolio belonging to an international insurance company operating in the Spanish market. In both lines we select claims at fault linked to civil liability coverage. Moreover, in the case of the auto insurance line we specifically differentiated two types of claims: only property damage and bodily injury. This distinction has been used previously in other studies focused on the severity of auto insurance claims (see Bahraoui et al., 2015; Bolancé et al., 2014; Bahraoui et al., 2014; Bolancé et al., 2008).

The rest of this paper is structured as follows: In Section 2, we review some univariate and trivariate mixed Poisson GLMs and introduce the main notation. In Section 3, we present the three mixed models which result in three trivariate Sarmanov with NB GLMs as marginal distributions. We analyse some properties and propose an algorithm for estimating the models based on the specificity of the Sarmanov distribution. In Section 4, we describe the data and discuss the results of the numerical application. Finally, we draw 
some conclusions in Section 5.

\section{Mixed Poisson distributions}

A mixed Poisson distribution is a generalization of the Poisson distribution that can overcome the restriction that the mean is equal to the variance, a restriction that is inappropriate for most counting random variables. A key property of this distribution is that it can be easily expressed as a GLM.

A well-known example of the mixed Poisson distribution is the NB distribution, which mixes the Poisson and Gamma distributions.

\subsection{Univariate case}

Let $N$ be the random variable (r.v.) total number of a certain type of claims of one insured for a given period. We assume that $N \sim \operatorname{Poisson}(\theta)$, where $\theta$ is the realization of a positive and continuous r.v. $\Theta$ having a probability density function (p.d.f.) $h$; hence, $N$ follows a mixed Poisson distribution with a probability function (p.f.) given by:

$$
\operatorname{Pr}(N=n)=\int_{0}^{\infty} e^{-\theta} \frac{\theta^{n}}{n !} h(\theta) d \theta .
$$

We recall that the expected value, variance and Laplace transform of this distribution are, respectively:

$$
\mathbb{E} N=\mathbb{E} \Theta, \operatorname{Var} N=\mathbb{E} \Theta+\operatorname{Var} \Theta, \mathscr{L}_{N}(t)=\mathbb{E}\left(e^{-t N}\right)=\mathscr{L}_{\Theta}\left(1-e^{-t}\right),
$$

where $\mathscr{L}_{\Theta}$ denotes the Laplace transform of $\Theta$.

In the following case, for sake of consistency with the GLMs, we shall consider the parameterization of the mixed Poisson distribution such that $\mathbb{E} N=\mu \theta$. Moreover, since in our numerical example we have three different types of claims, we shall index the r.v. $N$ with the index $j$ denoting the claims type wherever necessary.

\subsubsection{Negative Binomial case}

This distribution can be obtained by mixing Poisson and Gamma distributions. Hence, for consistency with the NB GLM, we assume that $N \sim$ Poisson $(\mu \theta)$, where $\mu>0$ is a fixed parameter and $\theta$ the realization of a Gamma distributed r.v. with mean 1 and variance $1 / \alpha$, i.e., $\Theta \sim \operatorname{Gamma}(\alpha, \alpha), \alpha>0$. We easily obtain that:

$$
\begin{aligned}
\operatorname{Pr}(N=n) & =\int_{0}^{\infty} e^{-\mu \theta} \frac{(\mu \theta)^{n}}{n !} h(\theta) d \theta \\
& =\frac{\Gamma(\alpha+n)}{n ! \Gamma(\alpha)}\left(\frac{\alpha}{\alpha+\mu}\right)^{\alpha}\left(\frac{\mu}{\alpha+\mu}\right)^{n}, n \in \mathbb{N}
\end{aligned}
$$


hence $N \sim N B(\alpha, \tau)$, where $\tau=\frac{\alpha}{\alpha+\mu}$. In this case, from the properties of the NB distribution we have that:

$$
\begin{aligned}
& \mathbb{E} N=\mu, \operatorname{Var} N=\mu+\frac{\mu^{2}}{\alpha}, \\
& \mathscr{L}_{N}(t)=\mathscr{L}_{\Theta}\left(\mu\left(1-e^{-t}\right)\right)=\left(\frac{\alpha}{\alpha+\mu\left(1-e^{-t}\right)}\right)^{\alpha}, t>\ln \frac{\alpha}{\alpha+\mu} .
\end{aligned}
$$

\subsubsection{Adding exposure and explanatory variables. GLMs}

Recall that in the numerical example we have different types of claim; hence, we let $N_{j}$ denote the r.v. total number of claims of type $j, j=1, \ldots, m$, where $m$ is the number of different claim types. At this point, we also introduce subscript $i$ related to individual $(i=$ $1, \ldots, I)$. We know that during the period analyzed, the policyholders could have contracted more than one policy in the same line and, furthermore, that the duration of one contract could be shorter than that of the period analyzed. This means that the policyholder's exposure to risk may differ. Let $E_{i j}$ be the exposure of individual $i$ in the contracted coverage $j$. We define $E_{i j}=1$ if the policyholder has contracted exactly one policy during the entire period under analysis; otherwise, we obtain $E_{i j}>1$ if the policyholder has contracted more than one policy and the total duration is longer than that of the period analyzed and, alternatively, we obtain $E_{i j}<1$ if the total duration is shorter than that of the period analyzed.

Additionally, we shall now consider the more general situation when the total number of a certain type of claim, $N_{i j}$, depends on certain individual characteristics of the policyholder $i$, i.e., we include explanatory variables (covariates) in GLM form. There are three components to GLM:

1. A stochastic component, which states that the observed r.v.s $N_{i j}$ are independent and distributed in the exponential family.

2. A systematic component, according to which a set of covariates $X_{i 0}, \ldots, X_{i p}$, where $X_{i 0}=1, \forall i$ is a constant term, produces a linear predictor with parameters $\beta_{0 j}, \ldots, \beta_{p j}$ for each observation, i.e., $\eta_{i j}=\sum_{k=0}^{p} X_{i k} \beta_{k j}$.

3. A link function $g$ relating the expected value of the stochastic component to the systematic component by $\eta_{i j}=g\left(\mu_{i j}\right)$, where $\mu_{i j}=\mathbb{E}\left(N_{i j}\right)$.

For counting variables, a Poisson GLM is the first choice, in which case the canonical link function is the logarithmic function, i.e., $\eta_{i j}=\ln \left(\mu_{i j}\right) \Leftrightarrow \mu_{i j}=\exp \left(\eta_{i j}\right)$. However, in practice, the Poisson GLM does not usually provides a good fit because of the overdispersion that occurs when the response variance is greater than the mean. Alternatively, NB GLMs have been developed using the same link function (see McCullagh and Nelder, 1989). 
Negative Binomial GLM with exposure. Such a model can be considered to arise wen we mix the Poisson distribution with a $\operatorname{Gamma}(\alpha, \alpha)$ distribution as in formula (3). We denote by $\beta_{0}$ the intercept coefficient and, in view of the numerical study, we shall also introduce the exposure (note that the exposure frequently appears in GLMs as weights).

Let $\mathbf{X}_{i}=\left(1, X_{i 1}, \ldots, X_{i p}\right)^{\prime}$ be a column vector with the values of the explanatory variables of individual $i$ and $\beta_{j}=\left(\beta_{0 j}, \beta_{1 j}, \ldots, \beta_{p j}\right)^{\prime}$ the parameters vector associated with the coverage $j$. We assume the logarithmic link function $\mathbf{X}_{i}^{\prime} \beta_{j}=\ln \left(\mu_{i j}\right)$ or, inversely, $\mu_{i j}=\exp \left(\mathbf{X}_{i}^{\prime} \beta_{j}\right)$; moreover, by including exposure $E_{i j}$, the individual expected value becomes:

$$
\mathbb{E}\left(N_{i j}\right)=E_{i j} \mu_{i j}=E_{i j} \exp \left(\mathbf{X}_{i}^{\prime} \beta_{j}\right) .
$$

Therefore, based on formula (3), we obtain for coverage $j$ (hence, with $\alpha_{j}$ denoting the Gamma parameter) of the $i$ th insured:

$$
\begin{aligned}
\operatorname{Pr}\left(N_{i j}=n\right) & =\frac{\Gamma\left(\alpha_{j}+n\right)}{n ! \Gamma\left(\alpha_{j}\right)}\left(\frac{\alpha_{j}}{\alpha_{j}+E_{i j} \mu_{i j}}\right)^{\alpha_{j}}\left(\frac{E_{i j} \mu_{i j}}{\alpha_{j}+E_{i j} \mu_{i j}}\right)^{n} \\
& =\frac{\Gamma\left(\alpha_{j}+n\right)}{n ! \Gamma\left(\alpha_{j}\right)} \frac{\alpha_{j}^{\alpha_{j}} \exp \left\{n\left(\ln \left(E_{i j}\right)+\mathbf{X}_{i}^{\prime} \beta_{j}\right)\right\}}{\left(\alpha_{j}+\exp \left\{\ln \left(E_{i j}\right)+\mathbf{X}_{i}^{\prime} \beta_{j}\right\}\right)^{\alpha_{j}+n}} .
\end{aligned}
$$

In this case, the likelihood function is:

$$
L\left(\alpha_{j}, \beta_{j}\right)=\prod_{i=1}^{I} \operatorname{Pr}\left(N_{i j}=n_{i j}\right)=\prod_{i=1}^{I} \frac{\Gamma\left(\alpha_{j}+n_{i j}\right)}{n_{i j} ! \Gamma\left(\alpha_{j}\right)} \frac{\alpha_{j}^{\alpha_{j}} \exp \left\{n_{i j}\left(\ln \left(E_{i j}\right)+\mathbf{X}_{i}^{\prime} \beta_{j}\right)\right\}}{\left(\alpha_{j}+\exp \left\{\ln \left(E_{i j}\right)+\mathbf{X}_{i}^{\prime} \beta_{j}\right\}\right)^{\alpha_{j}+n_{i j}}},
$$

where $n_{i j}$ is the number of observed claims of policyholder $i$ related to coverage $j$.

Also, the Laplace transform of $N_{i j}$ becomes:

$$
\begin{aligned}
\mathscr{L}_{N_{i j}}(t) & =\left(\frac{\alpha_{j}}{\alpha_{j}+E_{i j} \mu_{i j}\left(1-e^{-t}\right)}\right)^{\alpha_{j}} \\
& =\left(\frac{\alpha_{j}}{\alpha_{j}+\left(1-e^{-t}\right) \exp \left\{\ln \left(E_{i j}\right)+\mathbf{X}_{i}^{\prime} \beta_{j}\right\}}\right)^{\alpha_{j}} .
\end{aligned}
$$

\subsection{Multivariate case}

To obtain a multivariate mixed Poisson distribution, we let $N_{j} \sim$ Poisson $\left(\mu_{j} \theta\right)$ with $\mu_{j}>$ 0 fixed parameters, $j=1, \ldots, m$, and consider $\theta$ to be the realization of some positive r.v. $\Theta$ with pdf $h$. We also assume that, conditionally on $\Theta=\theta$, the r.v.s $N_{j}$ are independent. In the case of the numerical study, in what follows we shall only consider the NB case.

Multivariate Negative Binomial case. Under the assumptions outlined above, let 
$\Theta \sim \operatorname{Gamma}(\alpha, \alpha), \alpha>0$. In this case, the joint probabilities of $\mathbf{N}=\left(N_{1}, \ldots, N_{m}\right)$ are:

$$
\begin{aligned}
& \operatorname{Pr}(\mathbf{N}=\mathbf{n})=\int_{0}^{\infty} \operatorname{Pr}(\mathbf{N}=\mathbf{n} \mid \Theta=\theta) h(\theta) d \theta \\
= & \frac{\alpha^{\alpha}}{\Gamma(\alpha)}\left(\prod_{j=1}^{m} \frac{\mu_{j}^{n_{j}}}{n_{j} !}\right) \int_{0}^{\infty} \theta^{\sum_{j=1}^{m} n_{j}+\alpha-1} e^{-\theta\left(\sum_{j=1}^{m} \mu_{j}+\alpha\right)} d \theta \\
= & \frac{\Gamma\left(\alpha+\sum_{j=1}^{m} n_{j}\right)}{\Gamma(\alpha) \prod_{j=1}^{m} n_{j} !}\left(\frac{\alpha}{\alpha+\sum_{k=1}^{m} \mu_{k}}\right)^{\alpha} \prod_{j=1}^{m}\left(\frac{\mu_{j}}{\alpha+\sum_{k=1}^{m} \mu_{k}}\right)^{n_{j}}, \mathbf{n} \in \mathbb{N}^{m},
\end{aligned}
$$

which is the p.f. of the multivariate NB distribution defined as (see, e.g., Johnson et al., 1997):

$$
N B_{m}\left(\alpha ; \frac{\alpha}{\alpha+\sum_{j=1}^{m} \mu_{j}},\left(\frac{\mu_{j}}{\alpha+\sum_{j=1}^{m} \mu_{j}}\right)_{j=1, \ldots, m}\right) .
$$

For our numerical application, we shall need the trivariate NB distribution $(m=3)$, in which we also include the exposure of each individual; i.e., introducing the subscript $i$ related to the individual, we have that for $\mathbf{N}_{i}=\left(N_{i 1}, N_{i 2}, N_{i 3}\right), i=1, \ldots, I$,

$$
\mathbf{N}_{i} \sim N B_{3}\left(\alpha ; \frac{\alpha}{\alpha+\sum_{k=1}^{3}\left(E_{i k} \mu_{i k}\right)},\left(\frac{\left(E_{i j} \mu_{i j}\right)}{\alpha+\sum_{k=1}^{3}\left(E_{i k} \mu_{i k}\right)}\right)_{j=1,2,3}\right) .
$$

The correlation coefficient between two marginals for individual $i$ is:

$$
\operatorname{corr}\left(N_{i j}, N_{i k}\right)=\sqrt{\frac{E_{i j} \mu_{i j} E_{i k} \mu_{i k}}{\left(E_{i j} \mu_{i j}+\alpha\right)\left(E_{i k} \mu_{i k}+\alpha\right)}}, 1 \leq j<k \leq 3 .
$$

Let $\left(n_{i 1}, n_{i 2}, n_{i 3}\right)_{i=1}^{I}$ be a trivariate data sample with the corresponding exposures $\left(E_{i 1}, E_{i 2}, E_{i 3}\right)_{i=1}^{I}$ and we denote $\mu_{i}=\left(\mu_{i 1}, \mu_{i 2}, \mu_{i 3}\right), i=1, \ldots, I$. Then the likelihood function with exposure is

$L\left(\alpha, \mu_{1}, \ldots, \mu_{n}\right)=\prod_{i=1}^{I} \frac{\Gamma\left(\alpha+\sum_{j=1}^{3} n_{i j}\right)}{\Gamma(\alpha) \prod_{j=1}^{3} n_{i j} !}\left(\frac{\alpha}{\alpha+\sum_{k=1}^{3} \mu_{i k} E_{i k}}\right)^{\alpha} \prod_{j=1}^{3}\left(\frac{\mu_{i j} E_{i j}}{\alpha+\sum_{k=1}^{3} \mu_{i k} E_{i k}}\right)^{n_{i j}}$.

To estimate the parameters, we shall use the EM method proposed in Ghitany et al. (2012), which facilitates the ML estimation for multivariate mixed Poisson GLMs.

The classical multivariate NB model in (5) assumes that the dependence is based on a common random factor $\Theta$, an assumption that implies a lack of flexibility in the dependence structure. Shi and Valdez (2014) considered some alternative multivariate models based on the NB and copulae that allow for a generalization of the dependence structure. Alternatively, we shall study a different method for generalizing the dependence structure by using the Sarmanov distribution. 


\section{Models based on the trivariate Sarmanov distribution}

\subsection{Trivariate Sarmanov distribution}

This distribution can be defined in the discrete, as well as in the continuous case. In general, it is known (see Kotz et al., 2000) that a trivariate random vector $\mathbf{Y}$ follows a continuous trivariate Sarmanov distribution if its joint p.d.f. is given for $\mathbf{y} \in \mathbb{R}^{3}$ by:

$$
\begin{aligned}
h_{\text {Sarm }}(\mathbf{y}) & =\prod_{j=1}^{3} h_{j}\left(y_{j}\right) \\
& \times\left(1+\sum_{1 \leq j<k \leq 3} \omega_{j k} \phi_{j}\left(y_{j}\right) \phi_{k}\left(y_{k}\right)+\omega_{123} \phi_{1}\left(y_{1}\right) \phi_{2}\left(y_{2}\right) \phi_{3}\left(y_{3}\right)\right),
\end{aligned}
$$

where $\left(h_{j}\right)_{j=1}^{3}$ are the marginal pdfs, $\left(\phi_{j}\right)_{j=1}^{3}$ are bounded non-constant kernel functions and $\omega_{j k}, \omega_{123}$ are real numbers such that:

$$
\left\{\begin{array}{l}
\int_{\mathbb{R}} \phi_{j}(y) h_{j}(y)=0, \text { for } j=1,2,3 \\
1+\sum_{1 \leq i<j \leq 3} \omega_{j k} \phi_{j}\left(y_{j}\right) \phi_{k}\left(y_{k}\right)+\omega_{123} \phi_{1}\left(y_{1}\right) \phi_{2}\left(y_{2}\right) \phi_{3}\left(y_{3}\right) \geq 0, \forall \mathbf{y} \in \mathbb{R}^{3} .
\end{array}\right.
$$

The correlation coefficient between two marginal variables is related to the parameters $\omega_{j k}$ and the kernel functions $\phi_{j}$ by:

$$
\operatorname{corr}\left(Y_{j}, Y_{k}\right)=\omega_{j k} \frac{\mathbb{E}\left[Y_{j} \phi_{j}\left(Y_{j}\right)\right] \mathbb{E}\left[Y_{k} \phi_{k}\left(Y_{k}\right)\right]}{\sqrt{\operatorname{Var}\left(Y_{j}\right) \operatorname{Var}\left(Y_{k}\right)}} .
$$

Proposition 1 Concerning the parameter $\omega_{123}$, it holds that:

$$
\omega_{123}=\frac{\mathbb{E}\left[\prod_{j=1}^{3}\left(Y_{j}-\mathbb{E} Y_{j}\right)\right]}{\prod_{j=1}^{3} \mathbb{E}\left[Y_{j} \phi_{j}\left(Y_{j}\right)\right]} .
$$

Proof 1 Let $j_{3}=6-j_{1}-j_{2}$, then we can write:

$$
\begin{aligned}
& \mathbb{E}\left[\prod_{j=1}^{3}\left(Y_{j}-\mathbb{E} Y_{j}\right)\right]=\prod_{j=1}^{3} \int_{\mathbb{R}}\left(y_{j}-\mathbb{E} Y_{j}\right) h_{j}\left(y_{j}\right) d y_{j} \\
+ & \sum_{1 \leq j_{1}<j_{2} \leq 3} \omega_{j_{1} j_{2}} \prod_{k=1}^{2} \int_{\mathbb{R}} \phi_{j_{k}}\left(y_{j_{k}}\right)\left(y_{j_{k}}-\mathbb{E} Y_{j_{k}}\right) h_{j_{k}}\left(y_{j_{k}}\right) d y_{j_{k}} \int_{\mathbb{R}}\left(y_{j_{3}}-\mathbb{E} Y_{j_{3}}\right) h_{j_{3}}\left(y_{j_{3}}\right) d y_{j_{3}} \\
+ & \omega_{123} \prod_{j=1}^{3} \int_{\mathbb{R}} \phi_{j}\left(y_{j}\right)\left(y_{j}-\mathbb{E} Y_{j}\right) h_{j}\left(y_{j}\right) d y_{j} \\
= & \omega_{123} \prod_{j=1}^{3} \mathbb{E}\left[Y_{j} \phi_{j}\left(Y_{j}\right)\right],
\end{aligned}
$$

which easily yields the result. 
As for the form of the kernel functions $\phi_{j}$, several alternatives are used in the literature: for example, the Farlie-Gumbel-Morgenstern (FGM) distribution obtained for $\phi_{j}=1-2 F_{j}$, where $F_{j}$ is the cumulative distribution function of the marginal $Y_{j}$. Unfortunately, this FGM is restricted by the fact that the correlation coefficient of any two marginals cannot exceed $1 / 3$ in absolute value; hence, we do not consider it any further here. Another form of the kernel function is $\phi_{j}(y)=y-\mathbb{E} Y_{j}$, but this case usually involves truncated marginals to satisfy the conditions (8), which complicates computations. Therefore, here, we shall consider a third choice, which we call the exponential kernel, i.e., $\phi_{j}(y)=e^{-y}-\mathscr{L}_{Y_{j}}(1)$.

Since we shall work solely with nonnegative values, this last function $\phi_{j}(y)=e^{-y}-$ $\mathscr{L}_{Y_{j}}(1)$ will be bounded. Note that it is also decreasing; hence, we denote:

$$
\begin{aligned}
m_{j} & =\inf _{y \geq 0} \phi_{j}(y)=\phi_{j}(\infty)=-\mathscr{L}_{Y_{j}}(1), \\
M_{j} & =\sup _{y \geq 0} \phi_{j}(y)=\phi_{j}(0)=1-\mathscr{L}_{Y_{j}}(1), j=1,2,3 .
\end{aligned}
$$

Then the conditions (8) yield the restrictions:

$$
\begin{aligned}
1+\omega_{j k} \varepsilon_{j} \varepsilon_{k} & \geq 0,1 \leq j<k \leq 3, \\
1+\sum_{1 \leq j<k \leq 3} \omega_{j k} \varepsilon_{j} \varepsilon_{k}+\omega_{123} \varepsilon_{1} \varepsilon_{2} \varepsilon_{3} & \geq 0,
\end{aligned}
$$

where $\varepsilon_{j} \in\left\{m_{j}, M_{j}\right\}, j=1,2,3$. From these conditions we can deduce bounds for the parameters $\omega_{j k}$ and $\omega_{123}$.

In the discrete case, the joint probabilities of the trivariate Sarmanov distribution are given for $\mathbf{n} \in \mathbb{N}^{3}$ by

$$
\begin{aligned}
& \operatorname{Pr}_{\text {Sarm }}(\mathbf{N}=\mathbf{n})=\prod_{j=1}^{3} \operatorname{Pr}\left(N_{j}=n_{j}\right) \\
& \times\left(1+\sum_{1 \leq j<k \leq 3} \omega_{j k} \phi_{j}\left(n_{j}\right) \phi_{k}\left(n_{k}\right)+\omega_{123} \phi_{1}\left(n_{1}\right) \phi_{2}\left(n_{2}\right) \phi_{3}\left(n_{3}\right)\right) .
\end{aligned}
$$

In this paper, we compare three models (see below) based on the trivariate Sarmanov distribution. All three models have the same marginals, but different kernel functions, and hence a different dependence structure.

\subsection{Model I}

For each individual $i$, we shall consider that $\mathbf{N}_{i}$ follows the discrete trivariate Sarmanov distribution expressed in (13) with type (4) NB GLM distributed marginals and kernel functions of exponential type

$$
\phi_{i j}\left(n_{j}\right)=e^{-n_{j}}-\mathscr{L}_{N_{i j}}(1), j=1,2,3 .
$$


In the case of this expression, note that when we use GLM marginals in (13), the kernel functions depend on individual $i$ and, therefore, from conditions (11) and (12), we have to calculate different bounds of $\omega_{j k}$ and $\omega_{123}$ for each $i$ (the $\omega$ 's do not depend on individual $i$, but their limits do). In practice, we shall need to select the narrowest bounds.

\subsection{Model II}

We assume that $\mathbf{N}_{i}$ follows a trivariate Poisson distribution with independent marginals, which is mixed with a trivariate Sarmanov distribution with Gamma marginals, i.e. we assume that the dependence is given by the unobserved factor $\Theta_{j}, j=1,2,3$. Our model is a version of that proposed by Abdallah et al. (2016) for the bivariate case with a different parametrization. More specifically, we use $\operatorname{Gamma}\left(\alpha_{j}, \alpha_{j}\right)$ marginals for the Sarmanov distribution, and we extend the model to the trivariate case. Since we also need to introduce the exposure, the p.f. of the mixed distribution is obtained by solving the following triple integral:

$$
\operatorname{Pr}\left(\mathbf{N}_{i}=\mathbf{n}\right)=\int_{0}^{\infty} \int_{0}^{\infty} \int_{0}^{\infty}\left(\prod_{j=1}^{3} e^{-E_{i j} \mu_{i j} \theta_{j}} \frac{\left(E_{i j} \mu_{i j} \theta_{j}\right)^{n_{j}}}{n_{j} !}\right) h_{\text {Sarm }}\left(\theta_{1}, \theta_{2}, \theta_{3}\right) d \theta_{1} d \theta_{2} d \theta_{3},
$$

where $h_{\text {Sarm }}$ is given in (7) with $h_{j}$ the pdf of the mixing marginal r.v. $\Theta_{j} \sim \operatorname{Gamma}\left(\alpha_{j}, \alpha_{j}\right), j=$ $1,2,3$, and the kernel functions $\phi_{j}\left(\theta_{j}\right)=e^{-\theta_{j}}-\mathscr{L}_{\Theta_{j}}(1)$. Note that in this model, the original trivariate Poisson distribution corresponds to the independence case. Then the resulting p.f. $\operatorname{Pr}\left(\mathbf{N}_{i}=\mathbf{n}\right)$ is also of the Sarmanov type, but with more complex kernel functions, as can be seen from the following proposition.

Proposition 2 Under the above assumptions, it holds that the mixed distribution of $\mathbf{N}_{i}$ has the p.f.:

$$
\begin{aligned}
\operatorname{Pr}\left(\mathbf{N}_{i}=\mathbf{n}\right)= & \prod_{j=1}^{3} \operatorname{Pr}\left(N_{i j}=n_{j}\right) \\
& {\left[1+\sum_{1 \leq j_{1}<j_{2} \leq 3} \omega_{j_{1} j_{2}} \prod_{k=1}^{2}\left(\left(\frac{\alpha_{j_{k}}+E_{i j_{k}} \mu_{i j_{k}}}{\alpha_{j_{k}}+E_{i j_{k}} \mu_{i j_{k}}+1}\right)^{\alpha_{j_{k}}+n_{j_{k}}}-\left(\frac{\alpha_{j_{k}}}{\alpha_{j_{k}}+1}\right)^{\alpha_{j_{k}}}\right)\right.} \\
& \left.+\omega_{123} \prod_{j=1}^{3}\left(\left(\frac{\alpha_{j}+E_{i j} \mu_{i j}}{\alpha_{j}+E_{i j} \mu_{i j}+1}\right)^{\alpha_{j}+n_{j}}-\left(\frac{\alpha_{j}}{\alpha_{j}+1}\right)^{\alpha_{j}}\right)\right]
\end{aligned}
$$

where the marginals $N_{i j} \sim N B\left(\alpha_{j}, \tau_{i j}\right)$ with $\tau_{i j}=\frac{\alpha_{j}}{\alpha_{j}+E_{i j} \mu_{i j}}, j=1,2,3$.

Proof 2 We have:

$$
\begin{gathered}
\operatorname{Pr}\left(\mathbf{N}_{i}=\mathbf{n}\right)=\int_{0}^{\infty} \int_{0}^{\infty} \int_{0}^{\infty}\left(\prod_{j=1}^{3} e^{-E_{i j} \mu_{i j} \theta_{j}} \frac{\left(E_{i j} \mu_{i j} \theta_{j}\right)^{n_{j}}}{n_{j} !} h_{j}\left(\theta_{j}\right)\right) \\
\times\left(1+\sum_{1 \leq j_{1}<j_{2} \leq 3} \omega_{j_{1} j_{2}} \prod_{k=1}^{2}\left(e^{-\theta_{j_{k}}}-\mathscr{L}_{\Theta_{j_{k}}}(1)\right)+\omega_{123} \prod_{j=1}^{3}\left(e^{-\theta_{j}}-\mathscr{L}_{\Theta_{j}}(1)\right)\right) .
\end{gathered}
$$


Recalling that $\mathscr{L}_{\Theta_{j}}(1)=\left(\frac{\alpha_{j}}{\alpha_{j}+1}\right)^{\alpha_{j}}$ is the Laplace transform of the Gamma $\left(\alpha_{j}, \alpha_{j}\right)$ distribution, then we first evaluate:

$$
\begin{aligned}
& \int_{0}^{\infty} e^{-E_{i j} \mu_{i j} \theta_{j}} \frac{\left(E_{i j} \mu_{i j} \theta_{j}\right)^{n_{j}}}{n_{j} !} h_{j}\left(\theta_{j}\right)\left(e^{-\theta_{j}}-\mathscr{L}_{\Theta_{j}}(1)\right) d \theta_{j} \\
= & \int_{0}^{\infty} e^{-E_{i j} \mu_{i j} \theta_{j}} \frac{\left(E_{i j} \mu_{i j} \theta_{j}\right)^{n_{j}}}{n_{j} !} \frac{\alpha_{j}^{\alpha_{j}}}{\Gamma\left(\alpha_{j}\right)} \theta_{j}^{\alpha_{j}-1} e^{-\alpha_{j} \theta_{j}}\left[e^{-\theta_{j}}-\left(\frac{\alpha_{j}}{\alpha_{j}+1}\right)^{\alpha_{j}}\right] d \theta_{j} \\
= & \frac{\alpha_{j}^{\alpha_{j}}\left(E_{i j} \mu_{i j}\right)^{n_{j}}}{\Gamma\left(\alpha_{j}\right) n_{j} !}\left[\int_{0}^{\infty} \theta_{j}^{\alpha_{j}+n_{j}-1} e^{-\left(E_{i j} \mu_{i j}+\alpha_{j}+1\right) \theta_{j}} d \theta_{j}-\left(\frac{\alpha_{j}}{\alpha_{j}+1}\right)^{\alpha_{j}} \int_{0}^{\infty} \theta_{j}^{\alpha_{j}+n_{j}-1} e^{-\left(E_{i j} \mu_{i j}+\alpha_{j}\right) \theta_{j}} d \theta_{j}\right] \\
= & \frac{\Gamma\left(\alpha_{j}+n_{j}\right)}{\Gamma\left(\alpha_{j}\right) n_{j} !}\left(\frac{\alpha_{j}}{\alpha_{j}+E_{i j} \mu_{i j}}\right)^{\alpha_{j}}\left(\frac{E_{i j} \mu_{i j}}{\alpha_{j}+E_{i j} \mu_{i j}}\right)^{n_{j}}\left[\left(\frac{\alpha_{j}+E_{i j} \mu_{i j}}{\alpha_{j}+E_{i j} \mu_{i j}+1}\right)^{\alpha_{j}+n_{j}}-\left(\frac{\alpha_{j}}{\alpha_{j}+1}\right)^{\alpha_{j}}\right] \\
= & \frac{\Gamma\left(\alpha_{j}+n_{j}\right)}{\Gamma\left(\alpha_{j}\right) n_{j} !} \tau_{i j}^{\alpha_{j}}\left(1-\tau_{i j}\right)^{n_{j}}\left[\left(\frac{\alpha_{j}+E_{i j} \mu_{i j}}{\alpha_{j}+E_{i j} \mu_{i j}+1}\right)^{\alpha_{j}+n_{j}}-\left(\frac{\alpha_{j}}{\alpha_{j}+1}\right)^{\alpha_{j}}\right],
\end{aligned}
$$

where $\tau_{i j}=\frac{\alpha_{j}}{\alpha_{j}+E_{i j} \mu_{i j}}$. Inserting this formula into (14) we obtain the stated form of $\operatorname{Pr}\left(\mathbf{N}_{i}=\mathbf{n}\right)$, which is also of the Sarmanov type with $N B\left(\alpha_{j}, \tau_{i j}\right)$ marginals and kernel functions $\phi_{i j}\left(n_{j}\right)=\left(\frac{\alpha_{j}+E_{i j} \mu_{i j}}{\alpha_{j}+E_{i j} \mu_{i j}+1}\right)^{\alpha_{j}+n_{j}}-\left(\frac{\alpha_{j}}{\alpha_{j}+1}\right)^{\alpha_{j}}$ (it can be easily shown that $\mathbb{E}\left[\phi_{i j}\left(N_{i j}\right)\right]=$ $0, \forall i, j)$.

In the following result we present restrictions for the parameters $\omega_{j k}$ and $\omega_{123}$.

Proposition 3 Under the assumptions of Model II, the following conditions must be fulfilled for all $i=1, \ldots, I$ :

$$
\begin{aligned}
& \max _{1 \leq j<k \leq 3}\left\{\frac{-1}{M_{i j} M_{i k}}, \frac{-1}{m_{j} m_{k}}\right\} \leq \omega_{j k} \leq \min _{1 \leq j<k \leq 3}\left\{\frac{-1}{M_{i j} m_{k}}, \frac{-1}{m_{j} M_{i k}}\right\}, \\
& \max _{\substack{1 \leq j<k \leq 3 \\
h=6-j-k}}\left\{\frac{-1}{\prod_{l=1}^{3} M_{i l}}-\sum_{\substack{1 \leq l_{1}<l_{2} \leq 3 \\
l_{3}=6-l_{1}-l_{2}}} \frac{\omega_{l_{1} l_{2}}}{M_{i l_{3}}}, \frac{-1}{m_{j} m_{k} M_{i h}}-\frac{\omega_{j k}}{M_{i h}}-\frac{\omega_{j h}}{m_{k}}-\frac{\omega_{k h}}{m_{j}}\right\} \leq \omega_{123}, \\
& \omega_{123} \leq \min _{\substack{1 \leq j<k \leq 3 \\
h=6-j-k}}\left\{\frac{-1}{\prod_{l=1}^{3} m_{l}}-\sum_{\substack{1 \leq l_{1}<l_{2} \leq 3 \\
l_{3}=6-l_{1}-l_{2}}} \frac{\omega_{l_{1} l_{2}}}{m_{l_{3}}}, \frac{-1}{M_{i j} M_{i k} m_{h}}-\frac{\omega_{j k}}{m_{h}}-\frac{\omega_{j h}}{M_{i k}}-\frac{\omega_{k h}}{M_{i j}}\right\},
\end{aligned}
$$

where $m_{j}=-\left(\frac{\alpha_{j}}{\alpha_{j}+1}\right)^{\alpha_{j}}, M_{i j}=\left(\frac{\alpha_{j}+E_{i j} \mu_{i j}}{\alpha_{j}+E_{i j} \mu_{i j}+1}\right)^{\alpha_{j}}-\left(\frac{\alpha_{j}}{\alpha_{j}+1}\right)^{\alpha_{j}}, j=1,2,3, i=1, \ldots, I$, and, by convention, $\omega_{j k}=\omega_{k j}$.

Proof 3 The kernel function $\phi_{i j}(n)=\left(\frac{\alpha_{j}+E_{i j} \mu_{i j}}{\alpha_{j}+E_{i j} \mu_{i j}+1}\right)^{\alpha_{j}+n}-\left(\frac{\alpha_{j}}{\alpha_{j}+1}\right)^{\alpha_{j}}, n \in \mathbb{N}$, is bounded and decreasing in $n$, hence its maximum is $M_{i j}=\phi_{i j}(0)=\left(\frac{\alpha_{j}+E_{i j} \mu_{i j}}{\alpha_{j}+E_{i j} \mu_{i j}+1}\right)^{\alpha_{j}}-\left(\frac{\alpha_{j}}{\alpha_{j}+1}\right)^{\alpha_{j}}>$ 
0 and its infimum is $m_{j}=\phi_{i j}(\infty)=-\left(\frac{\alpha_{j}}{\alpha_{j}+1}\right)^{\alpha_{j}}<0, \forall i=1, \ldots, I$. Consequently, this type of kernel (8) also yields the restrictions (11)-(12), and from (11), we easily obtain the stated bounds of $\omega_{j k}$. Regarding $\omega_{123}$, (12) is equivalent to $\omega_{123} \varepsilon_{1} \varepsilon_{2} \varepsilon_{3} \geq-1-$ $\sum_{1 \leq j<k \leq 3} \omega_{j k} \varepsilon_{j} \varepsilon_{k}$, and by replacing each $\varepsilon_{j}$ with the current $m_{j}$ and $M_{i j}$, we obtain the result.

We note that, since the maximum $M_{i j}=\phi_{i j}(0)$ depends on the individual expected value, the intervals for the parameters $\omega_{j k}$ and $\omega_{123}$ can differ for each $i$; hence, in practice, we need to select the narrowest interval.

\subsection{Model III}

For this model, we let $\mathbf{N}_{i}$ follow a mixed discrete trivariate Sarmanov distribution with independent Gamma mixing distributions, i.e.,

$$
\operatorname{Pr}\left(\mathbf{N}_{i}=\mathbf{n}\right)=\int_{0}^{\infty} \int_{0}^{\infty} \int_{0}^{\infty} \operatorname{Pr}_{S a r m}\left(\mathbf{N}_{i}=\mathbf{n}\right)\left(\prod_{j=1}^{3} h_{j}\left(\theta_{j}\right)\right) d \theta_{1} d \theta_{2} d \theta_{3},
$$

where $\operatorname{Pr}_{\text {Sarm }}\left(\mathbf{N}_{i}=\mathbf{n}\right)$ is the discrete trivariate Sarmanov distribution (13) with Poisson marginals given by

$\operatorname{Pr}_{S a r m}\left(\mathbf{N}_{i}=\mathbf{n}\right)=\left(\prod_{j=1}^{3} e^{-E_{i j} \mu_{i j} \theta_{j}} \frac{\left(E_{i j} \mu_{i j} \theta_{j}\right)^{n_{j}}}{n_{j} !}\right)\left(1+\sum_{1 \leq j_{1}<j_{2} \leq 3} \omega_{j_{1} j_{2}} \phi_{i j_{1}}\left(n_{j_{1}}\right) \phi_{i j_{2}}\left(n_{j_{2}}\right)+\omega_{123} \prod_{j=1}^{3} \phi_{i j}\left(n_{j}\right)\right)$

and with kernel function $\phi_{i j}(n)=e^{-n}-\mathscr{L}_{P o\left(E_{i j} \mu_{i j} \theta_{j}\right)}(1)$. The mixing distributions $h_{j}$ are $\operatorname{Gamma}\left(\alpha_{j}, \alpha_{j}\right)$.

As can be seen from the following proposition, the resulting distribution is also of the Sarmanov type with the same marginals as in Model II, but with different kernel functions.

Proposition 4 Under the above assumptions, the p.f. of the mixed distribution of $\mathbf{N}_{i}$ is:

$$
\begin{aligned}
\operatorname{Pr}\left(\mathbf{N}_{i}=\mathbf{n}\right)= & \prod_{i=1}^{3} \operatorname{Pr}\left(N_{i j}=n_{j}\right)\left[1+\sum_{1 \leq j_{1}<j_{2} \leq 3} \omega_{j_{1} j_{2}} \prod_{k=1}^{2}\left(e^{-n_{j_{k}}}-\left(\frac{\alpha_{j_{k}}+E_{i j_{k}} \mu_{i j_{k}}}{\alpha_{j_{k}}+E_{i j_{k}} \mu_{i j_{k}}\left(2-e^{-1}\right)}\right)^{\alpha_{j_{k}}+n_{j_{k}}}\right)\right. \\
& \left.+\omega_{123} \prod_{j=1}^{3}\left(e^{-n_{j}}-\left(\frac{\alpha_{j}+E_{i j} \mu_{i j}}{\alpha_{j}+E_{i j} \mu_{i j}\left(2-e^{-1}\right)}\right)^{\alpha_{j}+n_{j}}\right)\right]
\end{aligned}
$$

where, as before, the marginals $N_{i j} \sim N B\left(\alpha_{j}, \tau_{i j}\right)$ with $\tau_{i j}=\frac{\alpha_{j}}{\alpha_{j}+E_{i j} \mu_{i j}}, j=1,2,3$. 
Proof 4 Since $\phi_{i j}(n)=e^{-n}-e^{-E_{i j} \mu_{i j} \theta_{j}\left(1-e^{-1}\right)}$, we obtain

$$
\begin{aligned}
& \int_{0}^{\infty} e^{-E_{i j} \mu_{i j} \theta_{j}} \frac{\left(E_{i j} \mu_{i j} \theta_{j}\right)^{n_{j}}}{n_{j} !} h_{j}\left(\theta_{j}\right) \phi_{i j}\left(n_{j}\right) d \theta_{j} \\
= & \int_{0}^{\infty} e^{-E_{i j} \mu_{i j} \theta_{j}} \frac{\left(E_{i j} \mu_{i j} \theta_{j}\right)^{n_{j}}}{n_{j} !} \frac{\alpha_{j}^{\alpha_{j}}}{\Gamma\left(\alpha_{j}\right)} \theta_{j}^{\alpha_{j}-1} e^{-\alpha_{j} \theta_{j}}\left[e^{-n_{j}}-e^{\left.-E_{i j} \mu_{i j} \theta_{j}\left(1-e^{-1}\right)\right] d \theta_{j}}\right. \\
= & \frac{\alpha_{j}^{\alpha_{j}}\left(E_{i j} \mu_{i j}\right)^{n_{j}}}{\Gamma\left(\alpha_{j}\right) n_{j} !} \int_{0}^{\infty}\left[\theta_{j}^{\alpha_{j}+n_{j}-1} e^{-\left(E_{i j} \mu_{i j}+\alpha_{j}\right) \theta_{j}} e^{-n_{j}}-\theta_{j}^{\alpha_{j}+n_{j}-1} e^{-\left(E_{i j} \mu_{i j}+\alpha_{j}+E_{i j} \mu_{i j}\left(1-e^{-1}\right)\right) \theta_{j}}\right] d \theta_{j} \\
= & \frac{\Gamma\left(\alpha_{j}+n_{j}\right)}{\Gamma\left(\alpha_{j}\right) n_{j} !}\left[e^{-n_{j}}\left(\frac{\alpha_{j}}{\alpha_{j}+E_{i j} \mu_{i j}}\right)^{\alpha_{j}}\left(\frac{E_{i j} \mu_{i j}}{\alpha_{j}+E_{i j} \mu_{i j}}\right)^{n_{j}}-\frac{\left.E_{i j}\right)^{n_{j}}}{\left(E_{i j} \mu_{i j}+\alpha_{j}+E_{i j} \mu_{i j}\left(1-e^{-1}\right)\right)^{\alpha_{j}+n_{j}}}\right] \\
= & \frac{\Gamma\left(\alpha_{j}+n_{j}\right)}{\Gamma\left(\alpha_{j}\right) n_{j} !} \tau_{i j}^{\alpha_{j}}\left(1-\tau_{i j}\right)^{n_{j}}\left[e^{-n_{j}}-\left(\frac{\alpha_{j}+E_{i j} \mu_{i j}}{\alpha_{j}+E_{i j} \mu_{i j}\left(2-e^{-1}\right)}\right)^{\alpha_{j}+n_{j}}\right] .
\end{aligned}
$$

Inserting this into (15), with some straightforward calculations, we obtain the stated formula.

The restrictions on the parameters $\omega_{j k}$ and $\omega_{123}$ are similar to these given in Proposition 3 with the maximums:

$$
M_{i j}=\phi_{i j}(0)=1-\left(\frac{\alpha_{j}+E_{i j} \mu_{i j}}{\alpha_{j}+E_{i j} \mu_{i j}\left(2-e^{-1}\right)}\right)^{\alpha_{j}} .
$$

However, in this case, the minimums

$$
m_{i j}=\min _{n_{j} \in \mathbb{N}}\left\{e^{-n_{j}}-\left(\frac{\alpha_{j}+E_{i j} \mu_{i j}}{\alpha_{j}+E_{i j} \mu_{i j}\left(2-e^{-1}\right)}\right)^{\alpha_{j}+n_{j}}\right\}
$$

also depend on individual $i$; moreover, they are obtained for some value in $\mathbb{N}$, and not by letting $n_{j} \rightarrow \infty$ as before.

\subsection{Estimation procedure for Models I, II and III}

Given the restricted shape of the parameters space, it is not easy to estimate all the parameters of Model I together. The same holds for the parameters of Model II and Model III. However, the specific shape of the Sarmanov distribution, which clearly splits into two parts -the marginal distributions and the dependence structure-, allows for the following approach:

- First, we estimate the parameters $\beta_{j}, j=1,2,3$, associated with the expected values, i.e., with $\mu_{i j}=\exp \left(\mathbf{X}_{i}^{\prime} \beta_{j}\right)$; the resulting estimations are denoted by $\hat{\beta}=\left(\hat{\beta}_{1}, \hat{\beta}_{2}, \hat{\beta}_{3}\right)$.

- Second, the parameters that define the variance and covariance matrix, i.e. $\alpha_{1}, \alpha_{2}$, $\alpha_{3}, \omega_{12}, \omega_{13}, \omega_{13}$ and $\omega_{123}$ can also be estimated. 
The parameters $\beta_{j}, j=1,2,3$ are estimated from the marginal distributions, i.e., we obtain $\hat{\beta}_{j}, j=1,2,3$ by maximizing the likelihood of the Poisson or NB GLM for each univariate marginal. If the NB model is the true one, both estimations are unbiased. From the ML estimations of the univariate NB GLM we also obtain the initial estimated values $\hat{\alpha}_{1}^{0}, \hat{\alpha}_{2}^{0}, \hat{\alpha}_{3}^{0}$, which are the starting values of the following iterative algorithm.

To estimate the dependence parameters, we define the following two conditional likelihoods: $L(\hat{\omega} \mid \hat{\alpha}, \hat{\beta})$ and $L(\hat{\alpha} \mid \hat{\omega}, \hat{\beta})$, where $\hat{\alpha}=\left(\hat{\alpha}_{1}, \hat{\alpha}_{2}, \hat{\alpha}_{3}\right)$ and $\hat{\omega}=\left(\hat{\omega}_{12}, \hat{\omega}_{13}, \hat{\omega}_{23}, \hat{\omega}_{123}\right)$ are two vectors with estimated parameters that allows us to obtain an estimation for the variance and covariance matrix.

The starting values for the dependence parameters, $\hat{\omega}^{0}=\left(\hat{\omega}_{12}^{0}, \hat{\omega}_{13}^{0}, \hat{\omega}_{23}^{0}, \hat{\omega}_{123}^{0}\right)$, are obtained by maximizing the first conditional likelihood given $\hat{\beta}$ and $\hat{\alpha}^{0}=\left(\hat{\alpha}_{1}^{0}, \hat{\alpha}_{2}^{0}, \hat{\alpha}_{3}^{0}\right)$. To this end, we need to define the parameters space (i.e., the current restrictions on the $\omega s$ ). To do this, we determine the signs of the parameters in $\hat{\omega}^{0}$ and their intervals (note that this procedure is general for any iteration $l$ in the following procedure). To find the signs, we use sample estimators based on (9) and (10). Taking these signs into account, we define variation intervals using Proposition 3. Starting with $l=0$, the rest of the procedure is divided in two steps:

- Step 1 (iteration $l$ ) Within the parameter space obtained based on the estimated signs and intervals (cf. to the procedure described above), find $\hat{\omega}^{l}$ by maximizing the conditional likelihood $L\left(\hat{\omega}^{l} \mid \hat{\alpha}^{l}, \hat{\beta}\right)$.

- Step 2 Obtain $\hat{\alpha}^{l+1}$ by maximizing the conditional likelihood $L\left(\hat{\alpha}^{l+1} \mid \hat{\omega}^{l}, \hat{\beta}\right)$.

If in Step 2 we have that $L\left(\hat{\alpha}^{l+1} \mid \hat{\omega}^{l}, \hat{\beta}\right) \leq L\left(\hat{\omega}^{l} \mid \hat{\alpha}^{l}, \hat{\beta}\right)$, we stop and consider the solution from the last iteration, Step 1; otherwise, we return to Step 1 for the next iteration.

Once we have estimated the parameters, we can calculate their standard errors using the approximate Hessian by Richardson's method implemented in R Software.

By way of alternative, in our algorithm, we also used an approach based on the EM algorithm proposed in Ghitany et al. (2012) to estimate new values of the $\beta_{j} \mathrm{~s}, j=1,2,3$ in Step 2 at each iteration. However, the results are practically the same and computational time did not inprove.

\section{Numerical Study}

We fitted the models presented above to a data set from a multinational insurance company. The data come from the Spanish insurance market and consist of a random sample of 162,019 policyholders who had had one or more auto and home policies during the decade 2006-2015. We used three dependent variables: the number of claims in auto insurance at fault involving only property damage (PD); the number of claims in auto insurance at fault with bodily injury (BI); and, the number of claims in home $(\mathrm{H})$ insurance 
at fault. In Table 1 we show the claims frequency for each type of risk. For BI the maximum number of claims reported by a policyholder was six. For PD and $\mathrm{H}$ this maximum value reached 40 and 23 , respectively.

In Table 2 we show four different statistics used to measure the dependence between the number of claims for each risk type. We also show the $p$-value associated with the significance test of each statistic. The statistics used were the following: chi-square (left upper triangle), to test for independence between discrete variables; the Pearson coefficient (left lower triangle), to test linear dependence; and, the Kendall and Spearman coefficients (right upper and lower triangles, respectively) to test non-linear dependence. To calculate the chi-square test statistic, we considered values of the number of claims from 0 to 6 or more and computed the $p$-values using the Monte Carlo method (see Hope, 1968).

From Table 2, note that all the statistics indicate that the different types of accident rate are dependent, with the exception of the Chi-square statistic for $\mathrm{BI}$ and $\mathrm{H}$.

Table 1: Claims frequency.

\begin{tabular}{|r|ccccccc|}
\hline Number of claims & 0 & 1 & 2 & 3 & 4 & 5 & $\geq 6$ \\
\hline \hline Auto Property Damage & 137437 & 15650 & 5247 & 1946 & 847 & 371 & 521 \\
\hline Auto Bodily Injury & 156928 & 4586 & 440 & 50 & 10 & 2 & 3 \\
\hline Home & 138694 & 17206 & 4125 & 1268 & 435 & 169 & 122 \\
\hline
\end{tabular}

Table 2: Dependence analysis ( $p$-value).

\begin{tabular}{|c|c|c|c|c|c|c|}
\hline & Chi-Squared Statistics ( $p-$ value) & & & Kendall & p-value) & \\
\hline & $\begin{array}{rr}\text { PD } & \text { BI }\end{array}$ & $\mathrm{H}$ & & PD & BI & $\mathrm{H}$ \\
\hline PD & $42462(0.0005)$ & $64.731(0.013)$ & PD & & $0.395(0.000)$ & $0.006(0.010)$ \\
\hline BI & $0.444(0.000)$ & $25.261(0.441)$ & BI & $0.403(0.000)$ & & $0.006(0.009)$ \\
\hline $\mathrm{H}$ & $0.006(0.003)$ & & $\mathrm{H}$ & $0.006(0.010)$ & $0.006(0.009)$ & \\
\hline & Pearson (p-value) & & & \multicolumn{2}{|c|}{ Spearman (p-value) } & \\
\hline
\end{tabular}

The explanatory variables (covariates) used are listed in Table 3 with their respective means and variances. The values of these variables correspond to the latest available information for each policyholder. Although the models allow different covariates associated with each dependent variable to be used, here we opted for the same covariates for all three dependent variables, choosing them in relation to the policyholders' characteristics. Among the explanatory variables, we included "Gender" (note that while in the Spanish insurance market this variable cannot be included to calculate the insurance premium, it should be considered in the risk analysis). We also delimited three zones as areas of residence. The first zone consists of the big cities, which in Spain correspond to Barcelona and Madrid; the second corresponds to the north, given its specific weather; while the third corresponds to the rest of the country, and is defined as the reference zone. 
Finally, we also included the age of the policyholder and the fact that the policyholder has contracted policies in other lines (e.g., accident insurance, life insurance, pension plans, etc.).

Table 3: Explanatory variables in the models (the values correspond to the latest available information for each policyholder).

\begin{tabular}{|c|l|r|r|}
\hline Variable & \multicolumn{1}{|c|}{ Description } & Mean & Variance \\
\hline$X_{1}$ & Gender of the policyholder: $=1$ if woman, =0 if man & 0.237 & 0.181 \\
$X_{2}$ & Area of residence: $=1$ if big city, =0 if other & 0.195 & 0.157 \\
$X_{3}$ & Area of residence: $=1$ if north, =0 if other & 0.291 & 0.206 \\
$X_{4}$ & Age of policyholder & 53.270 & 172.087 \\
$X_{5}$ & Client has other polices in the same company: $=1$ if yes, =0 if no & 0.220 & 0.433 \\
\hline
\end{tabular}

Table 4 presents the results for the estimated parameters of the trivariate NB GLM according to model (5), which includes the dependence between the numbers of claims in different types of insurance coverages. In Table 4 we have also included the estimated parameters obtained when fitting three independent univariate NB GLM distributions (i.e., the model with independent marginals). Then, in Table 5, we show the results for the estimated parameters of Models I, II and III, i.e., the three models based on the trivariate Sarmanov distribution. The Akaike information criterion (AIC) indicates that all the trivariate Sarmanov models improve the trivariate NB GLM model. Moreover, note that both Models II and III considerably improve Model I and, although the difference is small, Model II fits better than Model III. 


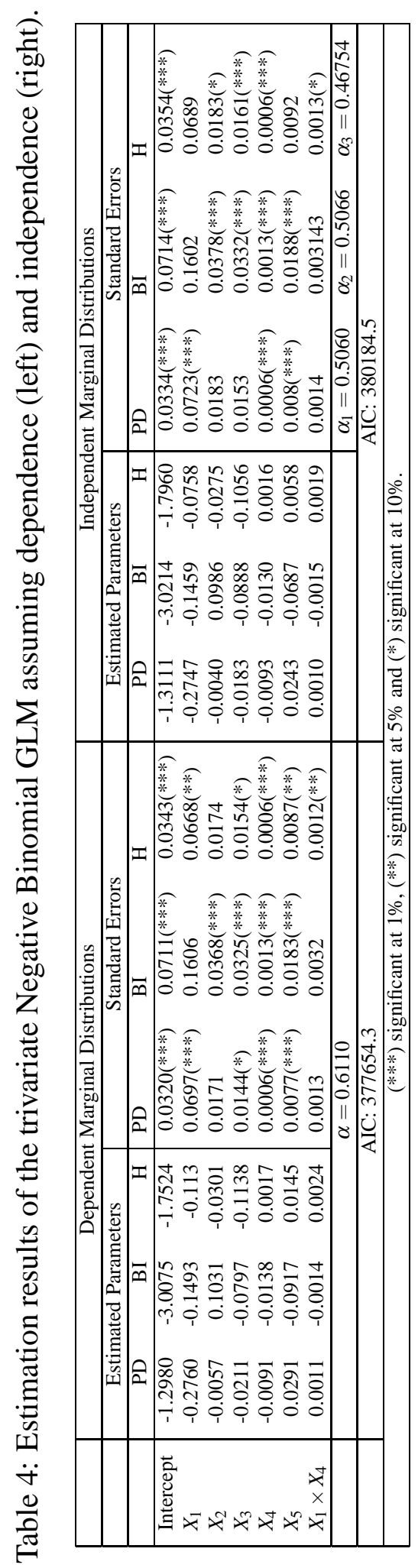




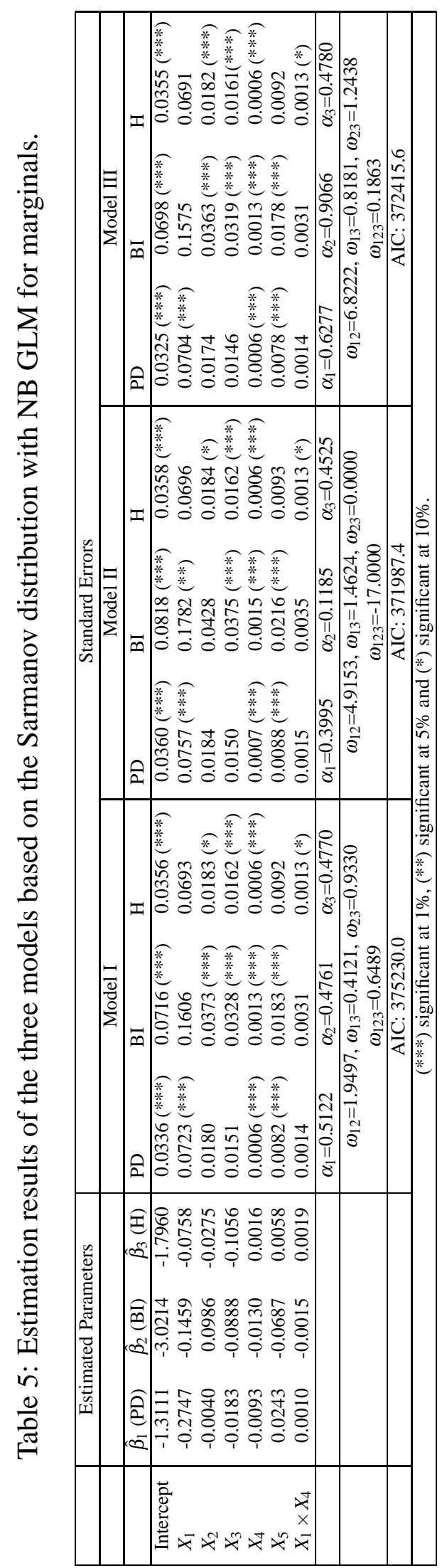


Note that, for the three Sarmanov models shown in Table 5, the values of the estimated parameters in the vectors $\hat{\beta}_{1}, \hat{\beta}_{2}$ and $\hat{\beta}_{3}$ associated with the covariates are the same, since they are obtained by ML estimation of univariate marginal NB GLMs. Moreover, these estimated parameters are very similar to those in the trivariate NB GLM presented in Table 4. The differences between the models are given by the values of parameters associated with the variance and covariance matrix of $\mathbf{N}_{i}=\left(N_{i 1}, N_{i 2}, N_{i 3}\right)$, which changes affecting the standard errors of the parameters in $\hat{\beta}_{1}, \hat{\beta}_{2}$ and $\hat{\beta}_{3}$. The trivariate NB GLMs with dependent marginals is the model for which most of these standard errors have the lowest values; consequently, this estimated model tends not to reject the individual significance of the estimated parameters associated with the explanatory variables.

A further difference between the four multivariate models estimated involves the dependence assumed between the coverages analyzed. Focusing on the Sarmanov models, we note that the values of the dependence parameters $\omega_{j k}$ differ considerably between models, and that this happens because each model is associated with a different dependency. More precisely, Model I assumes dependence between NB variables, Model II between Gamma variables and Model III between Poisson variables. To compare the dependence structures of the models, we calculated the correlation coefficient of each individual according to formulas (6) (for the trivariate NB model) and (9) (for the Sarmanov models) and then we calculated the mean of these individual correlations (see Table 6). It can be seen that the correlations estimated for Model II are the ones most similar to those observed in the data (see Table 2).

Table 6: Correlations deduced from the four trivariate models estimated.

\begin{tabular}{|c|c|c|c|}
\hline & \multicolumn{3}{|c|}{ Model I } \\
\hline & PD & $\mathrm{BI}$ & $\mathrm{H}$ \\
\hline PD & & 0.4562115 & 0.02477425 \\
\hline $\mathrm{BI}$ & 0.1102308 & & 0.02502437 \\
\hline \multirow[t]{4}{*}{$\mathrm{H}$} & 0.2284269 & 0.09967611 & \\
\hline & Trivariate $\mathrm{N}$ & ive Binomial & \\
\hline & \multicolumn{3}{|c|}{ Model II } \\
\hline & $\mathrm{PD}$ & BI & $\overline{\mathrm{H}}$ \\
\hline PD & & 0.5682647 & 0.02380325 \\
\hline $\mathrm{BI}$ & 0.827436 & & 0.01684441 \\
\hline \multirow[t]{2}{*}{$\mathrm{H}$} & 0.02585861 & 0.02475868 & \\
\hline & & III & \\
\hline
\end{tabular}

Even though Model II provides the best fit, all three models based on the Sarmanov distribution yield similar results with respect to the significance of the parameters $\hat{\beta}_{1}$, $\hat{\beta}_{2}$ and $\hat{\beta}_{3}$. These results indicate that the effect of the covariates depends on the type of coverage. For example, the effect of gender is negative and significant only in the case of property damage, i.e., women make less claims of this type. Living in big cities 
positively affects the number of bodily injury claims and negatively affects the number of home claims; however, living in the north of the country negatively affects both types of claims. The increasing age negatively affects the number of claims in the auto line and positively affects thats of claims in the home line. However, the parameter associated with the interaction of age and gender is significant at $10 \%$ only for the home line -the fact that this parameter is positive indicates that the effect of age is greater in the case of the women. Finally, the fact of having contracted more products with the same company only affects the auto line, where this effect is positive for property damage and negative for bodily injury.

\section{Conclusions}

In this paper, we have been able to identify the factors that affect each risk type by taking into account that the risks under analysis are dependent. To do this, we introduced three trivariate models with the same NB GLM marginals, but different dependence structures based on the Sarmanov distribution. Thus, where the first model (Model I) is simply a trivariate Sarmanov distribution with NB GLM marginals, the other two models where obtained by mixing three independent Poisson distributions with a Sarmanov distribution with Gamma distributed marginals (Model II) and a Sarmanov with Poisson marginals with independent Gamma distributions (Model III). These models were considered in connection with the number of claims made in three types of risks, two associated with the auto line (property damage and bodily insurance) and one associated with the home line.

Using a real data set from the Spanish insurance market, we also compared our proposed models with the trivariate NB GLM model and concluded that the two mixing models based on the Sarmanov distribution (Models II and III) improve the fit.

Moreover, we have proposed an algorithm for estimating the parameters in the Sarmanov based models. The expected number of claims estimated by each of the four models is practically the same. The main differences between the models are given by the values of the parameters associated with the dependence between the claims frequencies analyzed. These differences affect the risk quantification that depends on the correlation between the risk factors, and also the inference of the parameters associated with the covariates.

In conclusion, the mixing models based on the multivariate Sarmanov distribution add flexibility to the associated matrix of variances and covariances between dependent variables, resulting in a significant improvement in the fit compared to that obtained by simpler models including the multivariate NB GLM model and the multivariate discrete Sarmanov distribution with NB GLM marginals (Model I). 


\section{References}

Abdallah, A., Boucher, J., Cossette, H., 2016. Sarmanov family of multivariate distributions for bivariate dynamic claim counts model. Insurance: Mathematics and Economics 68, 120-133.

Bahraoui, Z., Bolancé, C., Pelican, E., Vernic, R., 2015. On the bivariate distribution and copula. an application on insurance data using truncated marginal distributions. Statistics and Operations Research Transactions, SORT 39, 209-230.

Bahraoui, Z., Bolancé, C., Pérez-Marín, A., 2014. Testing extreme value copulas to estimate the quantile. Statistics and Operations Research Transactions, SORT 38, 89102.

Bermudez, L., Karlis, D., 2011. Bayesian multivariate poisson models for insurance ratemaking. Insurance: Mathematics and Economics 48, 226-236.

Bolancé, C., Bahraoui, Z., Artís, M., 2014. Quantifying the risk using copulae with nonparametric marginal. Insurance: Mathematics and Economics 58, 46-56.

Bolancé, C., Guillén, M., Pinquet, J., 2003. Time-varying credibility for frequency risk models. Insurance: Mathematics and Economics 33, 273-282.

Bolancé, C., Guillén, M., Pinquet, J., 2008. On the link between credibility and frequency premium. Insurance: Mathematics and Economics 43,209-213.

Bolancé, C., Guillén, M., Pelican, E., Vernic, R., 2008. Skewed bivariate models and nonparametric estimation for cte risk measure. Insurance: Mathematics and Economics 43, 386-393.

Boucher, J.P., Denuit, M., Guillen, M., 2007. Risk classification for claim counts: A comparative analysis of various zero-inflated mixed Poisson and hurdle models. North American Actuarial Journal 11, 110-131.

Boucher, J.P., Inoussa, R., 2014. A posteriori ratemaking with panel data. ASTIN Bulletin 44, 587-612.

Brockett, P.L., Golden, L., Guillén, M., Nielsen, J., Parner, J., Pérez-Marín, A., 2008. Survival analysis of a household portfolio of insurance policies: how much time do you have to stop total customer defection? Journal of Risk and Insurance 75, 713-737. 
Frees, E., 2009. Regression Modelling with Actuarial and Financial Applications. Cambridge University Press.

Ghitany, M., Karlis, D., D.K., A.M., Al-Awadhi, F., 2012. An EM algorithm for multivariate mixed Poisson regression models and its application. Applied Mathematical Sciences 6, 6843-6856.

Guelman, L., Guillén, M., 2014. A causal inference approach to measure price elasticity in automobile insurance. Expert Systems with Applications 41, 387-396.

Guelman, L., Guillén, M., Pérez-Marín, A., 2014. A survey of personalized treatment models for pricing strategies in insurance. Insurance: Mathematics and Economics 58, $68-76$.

Guillén, M., Nielsen, J., Scheike, T., Pérez-Marín, A., 2012. Time-varying effects in the analysis of customer loyalty: A case study in insurance. Expert Systems with Applications 39, 3551-3558.

Hope, A.C.A., 1968. A simplified Monte Carlo significance test procedure. Journal of the Royal Statistical Society. Series B 30, 582-598.

Johnson, N., Kotz, S., Balakrishnan, N., 1997. Discrete multivariate distributions. Wiley.

Kotz, S., Balakrishnan, N., Johnson, N., 2000. Continuous Multivariate Distributions. Vol.1: Models and Applications. Wiley.

McCullagh, P., Nelder, J.A., 1989. Generalized linear models. Vol.37. CRC Press.

Pinquet, J., Guillén, M., Bolancé, C., 2001. Long-range contagion in automobile insurance data: estimation and implications for experience rating. ASTIN Bulletin 31, 337348.

Shi, P., Valdez, E., 2014. Multivariate negative binomial models for insurance claim counts. Insurance: Mathematics and Economics 55, 18-29. 


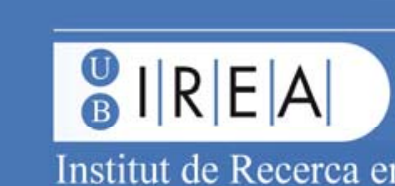

